\title{
Dissociating the Effects of Automatic Activation and Explicit Expectancy on Reaction Times in a Simple Associative Learning Task
}

\author{
Pierre Perruchet \\ Université de Bourgogne
}

\author{
Axel Cleeremans \\ Université libre de Bruxelles
}

\author{
Arnaud Destrebecqz \\ Université de Bourgogne
}

\begin{abstract}
After repeated associations between two events, E1 and E2, responses to E2 can be facilitated either because participants consciously expect E2 to occur after E1 or because E1 automatically activates the response to E2, or because of both. In this article, the authors report on 4 experiments designed to pit the influence of these 2 factors against each other. The authors found that the fastest responses to a target in a reaction time paradigm occurred when automatic activation was highest and conscious expectancy lowest. These results, when considered together with previous findings indicating that, under most conditions, the relation between expectancy and reaction times is in the opposite direction, are indicative of a reversed association - an interaction pattern that J. C. Dunn and K. Kirsner (1988) demonstrated to be the only one that unambiguously points to the involvement of independent processes.
\end{abstract}

Keywords: learning, conditioning, expectancy, automatism, dissociations

When two normatively unrelated events, E1 and E2, are repeatedly displayed in close temporal succession, the presentation of E1 modifies (and generally improves) the behavioral response to E2. This phenomenon has been investigated in several independent areas of research, such as associative priming (where E1 is the prime, and E2 is the target), classical conditioning (where E1 is the conditioned stimulus [CS], and E2 is the unconditioned stimulus [US]), and studies of motor behavior (where E1 is the warning stimulus, and E2 is the imperative stimulus in a reaction time paradigm). In each case, and even though different terminology has often been used, the same distinction between two general

Pierre Perruchet and Arnaud Destrebecqz, Laboratore d'Etude de L'apprentissage et du Developpement and Centre National de la Recherche Scientifique, Université de Bourgogne, Dijon, France; Axel Cleeremans, Cognitive Science Research Unit, Université libre de Bruxelles, Brussels, Belgium.

Arnaud Destrebecqz is now at the Cognitive Science Research Unit, Université libre de Bruxelles, Brussels, Belgium.

Axel Cleeremans is a Senior Research Associate of the National Fund for Scientific Research (Belgium). Arnaud Destrebecqz was supported by a post-doctoral grant from the Fyssen Foundation. This work was supported by grants from the Centre National de la Recherche Scientifique (Unité Mixte de Recherche, 5022), the Université de Bourgogne, the Région de Bourgogne (Apprentissage, Acquisition, Formation, Education), the European Commission (Grant HPRN-CT-1999-00065), and the Université Libre de Bruxelles.

We thank John Dunn, Sander Los, David Shanks, and Jennifer Stolz for their comments on an earlier version of the article. We also thank Delphine Malbec, Victorien Devessiere, and Eimeric Hivert for their assistance in running the experiments.

Correspondence concerning this article should be addressed to Pierre Perruchet, Université de Bourgogne, LEAD/CNRS, pôle AAFE, Esplanade Erasme, 21000, Dijon, France. E-mail: pierre.perruchet@u-bourgogne.fr classes of interpretations has been proposed. The first focuses on the conscious expectancy for E2 that is initiated by the occurrence of E1. The second class of interpretations posits some form of automatic activation through which the occurrence of E1 facilitates the response to E2 as a mandatory consequence of their having been repeatedly associated in the past. In this context, automatic activation is therefore assumed to reflect previous experience with the association, independent of the agent's conscious expectancy for E2. ${ }^{1}$

Expectancy and automatic activation are difficult to dissociate, because typical experimental manipulations would lead one to predict identical effects irrespective of whether they are assumed to depend on one or on the other of the two putative processes. For instance, both expectancy and automatic activation are likely to be influenced in the same manner by the number of previously experienced E1-E2 pairings (i.e., they would both increase). It is possible, however, to induce conscious expectancy while suppressing the influence of automatic activation altogether by verbally informing participants about the E1-E2 association without actually presenting such pairings. Using such a design, Epstein and Roupenian (1970) found that responses were almost identical to those induced by actual presentation of the pairings. These and other findings have resulted in the widespread endorsement of expectancy-based accounts of facilitation effects, both for conditioning (e.g., Bolles, 1972; Tolman, 1932) and for reaction time

\footnotetext{
${ }^{1}$ Throughout this article, the word expectancy is taken as identical to conscious expectancy, such as it can be measured through a subjective rating scale. The notion of unconscious expectancy is sometimes advocated to account for variations in reaction times or event-related potentials that cannot be attributed to variations in conscious expectancies (e.g., Matt, Leuthold, \& Sommer, 1992). We refer here to automatic effects to designate those factors.
} 
studies (e.g., Hale, 1967; Niemi \& Näätänen, 1981; Requin, Brener, \& Ring, 1991).

In contrast, inducing automatic activation while preventing the occurrence of conscious expectancy appears to be much more problematic. Prior attempts to do so have relied on situations in which participants were assumed to be unconscious of the E1-E2 associations. However, assessing the extent to which participants remain unaware of some regularity is notoriously difficult (Shanks \& St. John, 1994) and presents complex methodological and conceptual challenges. Awareness cannot be simply turned off, and regardless of the amount of care taken to prevent participants from consciously appreciating the situation, it seems that obtaining genuine demonstrations of unconscious influence with normal participants will always remain an elusive goal. As a consequence, the possibility of obtaining an associative response to E2 while participants are unaware of the E1-E2 association has been heavily challenged (e.g., Brewer, 1974; Lovibond \& Shanks, 2002). This state of affairs therefore leaves us in a situation in which the only mechanism through which E1 can influence the processing of E2 is one that is assumed to be mediated by conscious expectancy.

Rather than attempt to selectively prevent the occurrence of conscious expectancy, a better strategy to demonstrate the genuine influence of automatic activation might consist of pitting the two processes against each other. Perruchet (1985) showed how this can be achieved by manipulating the sequential structure of the material. In Perruchet's paradigm, E1 is followed by E2 in only $50 \%$ of the trials, on a random basis. This design naturally results in the production of both E1-alone sequences and of sequences of E1-E2 pairings of various lengths. Each trial may thus be defined by the nature and the length of the preceding run (i.e., a sequence of $1,2, \ldots n$ identical trials).

To see why this simple manipulation is relevant to the issues at hand, consider how conscious expectancy changes over trials with such stimulus material. In keeping with the so-called "gambler's fallacy" (N. H. Anderson, 1960; Burns \& Corpus, 2004; Jarvik, 1951; Keren \& Lewis, 1994) - that is, the belief that alternations should occur more frequently than repetitions in random sequences of events-people's expectancy for E2 should be high after a run of E1-alone events and low after a run of E1-E2 pairings. Further, this difference in degree of expectation should increase as a function of the length of the preceding run. If these assumptions are correct, we would therefore expect to observe that conscious expectancy for E2 is highest after a long run of E1 alone events and lowest after a long run of E1-E2 pairings. It should further decrease monotically between these two extreme points. This relationship is depicted in Figure 1, in which expectancy for E2 is represented as a function of run length in the various conditions, as labeled on the $x$-axis of the graph.

Let us now consider the degree of automatic activation caused by the anticipatory signal. The degree of automatic activation, as defined above, can be assumed to vary as a function of the strength of the E1-E2 association. The well-documented laws of associative learning and memory allow clear-cut predictions to be made about the effects of changes in the strength of the association between E1 and E2 in this situation. Indeed, one can expect that any pairing between E1 and E2 will strengthen the formation of an associative link between the two events, whereas any presentation of E1 alone should promote the extinction of the association. These laws have been known since Pavlov's day and have since been
Expected magnitude of the response to E2

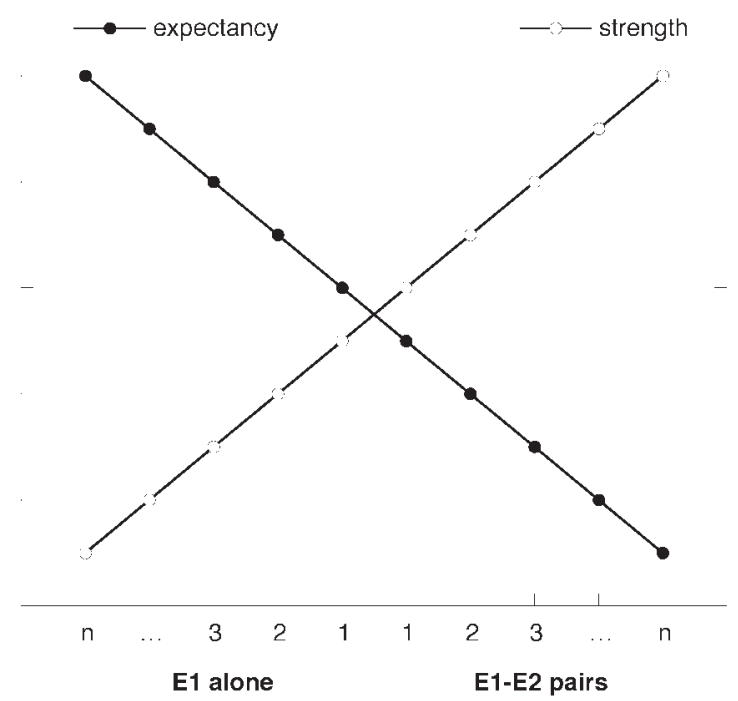

Figure 1. Let us consider a sequence composed of the repetition of a given event (E1) randomly paired on half of the trials with a second event (E2). Any trial of the sequence immediately follows either a run of 1, 2, 3, $\ldots n, \mathrm{E} 1$ alone or a run of $1,2,3, \ldots n, \mathrm{E} 1-\mathrm{E} 2$ pairs. The figure indicates that the nature of the preceding run ( $x$-axis) should have opposite effects on the conscious expectancy of E2 and on the strength of the E1-E2 association.

unambiguously and repeatedly confirmed in both the conditioning and the associative memory literatures (e.g., J. R. Anderson, 2000). Hence, associative strength should increase after a run of E1-E2 pairings and decrease after a run of E1-alone events. Further, this effect should be all the more marked the longer the runs are. Thus, on the basis of how associative strength changes when the run of events that precedes the current trial changes from a long series of E1-alone events to a long series of E1-E2 pairings, as labeled on the $x$-axis of Figure 1, we would expect an increasing monotonic trend. Note that, remarkably, this prediction is exactly opposite to what one would anticipate on the basis of conscious expectancy.

Perruchet (1985) implemented this paradigm in the context of a classical conditioning situation. In that study, E1 was a tone, and E2 was an air puff directed toward the participant's cornea. In classical conditioning, E1, which is initially neutral with respect to the reaction elicited by E2, ultimately comes to elicit this reaction after having been repeatedly coupled with E2. Perruchet confirmed that the tones eventually condition eyeblink responses. He also observed that participants' expectancies, which were assessed through a subjective rating scale administered before the onset of each trial, indeed followed the gambler's fallacy effect. The interesting point was that the probability of conditioned eyeblinks, when plotted as a function of the conditions represented on the $x$-axis of Figure 1, clearly followed an increasing linear trend in two separate experiments. In other words, the probability of conditioned responses matched almost perfectly what one would have expected on the basis of the traditional strength theory of conditioning (e.g., Bush \& Mosteller, 1951; Hull, 1943). Crucially, this pattern of responses cannot be attributed to concurrent variations in expectancy, as those variations would have induced the opposite trend. 
Bonic (1989) and Clark, Manns, and Squire (2001) replicated Perruchet's (1985) study and reported, just as in the initial study, a negative relationship between the probability of eyeblink conditioned response and subjective expectancy for the air puff-just as would have been expected on the basis of the notion that performance depends only on the strength of the E1-E2 association.

These results are important because they offer evidence for a mechanism that generates a direct link between E1 and E2, independent of participants' conscious expectancy: Participants verbally indicate that they expect the next event to not be followed by an air puff, yet it is precisely under those circumstances that their eyeblink response to the conditioned stimulus is strongest. In their critical overview on the role of awareness in classical conditioning, Lovibond and Shanks (2002) pointed out that "Perruchet's (1985) study provides the strongest evidence to date for a dissociation between eyeblink conditioning and expectancy; and his design provides a strong model for future investigations" (p. 8).

The main goal of the current study was to explore what happens when E2 is an imperative stimulus for a voluntary reaction instead of a US, such as the air puff used in Perruchet (1985) and Clark et al. (2001). Do reaction times (RTs) to E2 reflect participants' conscious expectations? Or do they instead vary in the same manner as conditioned eyeblink responses do in responses to changes in run length, that is, in complete opposition to subjective expectancy? This latter outcome may be anticipated if one takes it for granted that, when the unconditioned stimulus of a classical conditioning procedure is replaced by an imperative stimulus, RTs follow the same laws (repetition, extinction, inhibition, and so on) as classically conditioned reactions, as claimed long ago (Razran, 1936; Rexroad, 1936; Stephens, 1937; see also Los, Knol, \& Boers, 2001).

Because RTs may be sensitive to a larger variety of factors than the occurrence of eyeblink responses, shifting from conditioned reactions to RT measures raises the question of whether RTs could be inadvertently influenced by factors other than those of interest. Of relevance here is the research on sequential effects in random serial RT tasks. In those tasks, two stimuli, each associated with a specific response, appear in close succession on a random basis. A large number of studies have explored how performance on a given trial depends on the nature of the preceding sequence. Two effects have been reported. With very short response-stimuli intervals (RSI), a repetition effect is generally observed, whereby RTs for repetitions of the same stimulus are found to be shorter than RTs for alternations between the two stimuli (Bertelson, 1961). Most authors agree to ascribe this effect to some kind of automatic priming. With longer RSIs, RTs for alternation are typically shorter than RTs for repetition-an effect that is generally attributed to the involvement of strategic processes (e.g., Soetens \& Notebaert, 2005).

It is worth stressing that, despite some surface similarity with the issue we outlined above, the processes at play in serial RT tasks are in principle different. Whereas our focus is on the processes that mediate associative learning, serial RT tasks do not trigger the formation of associative links. For instance, in the terminology used in this article, the repetition priming effect concerns the influence of E2 on the processing of the next occurrence of E2, whereas our concern is about the effect of E1 on E2 as a result of prior E1-E2 pairings. However, the literature on serial RT tasks cannot be ignored, because the effects it reveals could be a source of confounding factors. Indeed, it turns out that the alternation of runs of E1-alone and E1-E2 pairings, which lies at the heart of our design, is partially confounded with the distribution of the temporal intervals between successive E2 events. Thus for instance, an E2 event that occurs after a long series of E1-alone trials has not been experienced for a long period; and conversely, when E2 occurs after a long series of E1-E2 pairings, it has been experienced several times in the recent past. In other words, the lag that separates successive occurrences of E2 is, by design, partially confounded with the nature of the runs of trials presented during the experiment.

In this context, and given our primary interest in revealing the effect of automatic associative activation, it appears especially important to avoid any confusion between this effect and the automatic priming that is due to stimulus repetitions. Bertelson (1961) noted that the repetition effect, which was observed to occur with a 50-ms-long RSI, disappeared when the RSI was increased to $500 \mathrm{~ms}$. Subsequent studies showed that the repetition effect can also be observed with longer RSIs when stimulusresponse compatibility is lowered, when the number of alternatives is increased (e.g., Bertelson, 1963; Bertelson \& Renkin, 1966; Vervaek \& Boer, 1980; see also Spence, Nicholls, \& Driver, 2001, for similar effects with intertrial intervals [ITIs] in the range of 1,500-1,900 ms), or when children rather than adults perform the task (e.g., Smulders et al., 2005). There is converging evidence, however, that the repetition effect is limited to RSIs of a very few seconds at best, regardless of paradigms and participants. When the RSI is longer than a few seconds, all results show shorter RTs for alternating stimuli and longer RTs for repeated stimuli (e.g., Hale, 1967; Hyman, 1953; see also a review in Kornblum, 1973). In the following studies, we designed the experimental conditions so as to prevent the influence of stimulus repetition. We achieved this by using ITIs that far exceed those for which a repetition effect has been observed (mean ITI $=10 \mathrm{~s}$ in Experiment 1 and $8 \mathrm{~s}$ in the subsequent experiments). ${ }^{2}$ The occurrence of repetition priming was made even more unlikely by the simplicity of our task and by our use of adults as participants. Finally, the possibility of biases because of the influence of nonassociative factors was further tested and ruled out in Experiment 4.

\section{Experiment 1}

\section{Method}

Participants. Twenty right-handed university students majoring in psychology agreed to act as subjects.

Materials. E1 was a $500-\mathrm{Hz}, 800-\mathrm{ms}$ tone, and E2 was a $2-\times 2-\mathrm{cm}$ white square displayed on a computer screen. Half of the trials consisted of a single tone, and half consisted of a tone-square pair. The sequence was

\footnotetext{
${ }^{2}$ In standard studies on serial reaction tasks, participants have to make a response on each trial, so that the most typical measure of the interval between trials is the time between the response and the onset of the next stimulus (RSI). This value is roughly similar to (although obviously shorter than) the ITI, that is, the time separating the onsets of two successive stimuli. However, the notion of RSI and ITI needs to be carefully distinguished in our study, in which no response is required on half of the trials. The ITI is defined here as the interval between the onset of successive E1, whether or not E1 is followed by E2. On the average, the mean RSI is twice as large as the mean ITI.
} 
constructed on the basis of the randomization with restriction method described by Nicks (1959). Random drawings were taken from a set of runs (and not a set of trials), the number and length of which had previously been computed. Because the longest run was set to five trials for the current experiments, the stimulus material included 1 run of five trials, 2 runs of four trials, 4 runs of three trials, 8 runs of two trials, and 16 runs of one trial. If $n$ designates the number of runs, and $l$ designates their length, then

$$
\sum_{i=1}^{s} n_{i} l_{i}=57
$$

is the total number of trials of each type (tones alone or tone-square pairings). The only way in which we modified Nicks's method was to add two pairings, one after the longest run of tone-square pairings and the other at the very end of the experiment. This made it possible to obtain an RT corresponding to the respective preceding runs. Thus the total number of trials experienced by subjects was $(57 \times 2)+2$, that is, a total of 116 trials. A different sequence was generated anew for each subject.

We collected expectancies for E2 with a linear potentiometer, using a slider moving from front to back with a maximum displacement of $4.5 \mathrm{~cm}$. The slider position was displayed on the screen as a cursor moving continuously on a horizontal scale $6 \mathrm{~cm}$ in length. The right end of the scale (marked "+") corresponded to the forward position of the slider and was used to indicate maximum expectancy. The left end of the scale (marked "-") corresponded to the back position of the slider and was used to indicate that participants were certain that the white square would not occur on that particular moment. The white square appeared at the current position of the cursor on the screen.

Procedure. Following common practice in classical conditioning research (e.g., Knight, Nguyen, \& Bandettini, 2003), participants were instructed to continuously update the position of the cursor so as to reflect their current expectancy for the occurrence of the square. They did so by moving the slider with their left hand. They were also asked to press the space bar of the keyboard with their right hand as quickly as possible following the appearance of the square. The white square disappeared from the screen as soon as the space bar had been pressed. Participants were told that the white square would be displayed after only half of the tones, so as to prevent a strategy consisting of pressing the space bar in response to the tones, that is, in anticipation of the occurrence of the white square. The tone-square stimulus onset asynchrony (SOA) was $500 \mathrm{~ms}$, and the ITI (between the onset of two successive tones) varied randomly between 6 and $14 \mathrm{~s}(M=10 \mathrm{~s})$.

Data analysis. The position of the cursor (conscious expectancy rating) was converted with a 200-point scale and was recorded $450 \mathrm{~ms}$ after the onset of the tone, that is, $50 \mathrm{~ms}$ before the (possible) occurrence of the white square. RTs shorter than $100 \mathrm{~ms}$ and RTs longer than $900 \mathrm{~ms}$ were discarded and replaced by the subject's mean score. These outliers represented $1.14 \%$ and $1.58 \%$ of the RTs, respectively. Each of the measures was then ascribed to its appropriate condition, as labeled on the $x$-axis of Figure 1 (e.g., the scores of expectancy and the RTs taking into account the run length of two events comprised the values collected after a run of two events, as well as the values collected after the second trial of the runs consisting of three, four, and five events).

\section{Results and Discussion}

As shown in Figure 2, expectancy (left $y$-axis) for the white square differed, $F(9,171)=10.63, p<.001, M S E=2,002.93$, as a function of the length and nature of the preceding run. Expectancy of the target after a single tone-alone event is lower than after a single tone-square pairing, which suggests that subjects expected a repetition to occur after the presentation of a single trial of a given type. This phenomenon is known in the probability learning

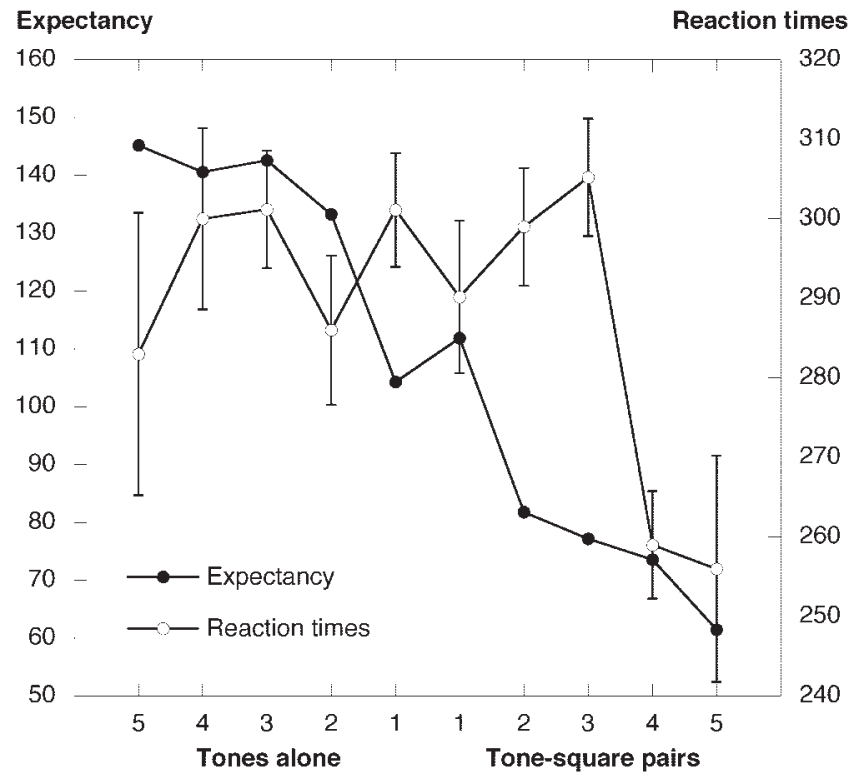

Figure 2. Mean conscious expectancy ratings (on a 200-point scale, left $y$-axis), and mean reaction times for E2 (right $y$-axis) as a function of the preceding run of trials (Experiment 1). The errors bars (available only for RTs because of computer failure) indicate standard errors.

literature as a positive recency effect. However, by and large, mean expectancy follows fairly well the decreasing linear trend, $F(1$, 171) $=89.34, p<.001, M S E=2,002.93$, that had been anticipated: Participants clearly exhibit lowered expectation that the tone will be followed by the white square after a series of recent occurrences of the pairing.

Mean RTs (right $y$-axis) appear on the same Figure 2 for easy comparison with expectancy. The means differed significantly, $F(9,171)=6.24, p<.001, M S E=999.28$, across conditions More interesting, the overall slope was negative, $F(1,19)=7.26$, $p=.014, M S E=1,939.22$, thus indicating that RTs improved while conscious expectancy decreased. However, the quadratic trend was also significant, $F(1,19)=14.84, p<.001, M S E=$ 1,432.82. An examination of Figure 2 shows that RTs for runs of four and five tone-square pairings were shorter than RTs for other runs, which did not differ from each other.

Overall, this experiment provides preliminary evidence that RTs are more sensitive to the automatic activation generated by the preparatory signal than to conscious expectancy of the target. However, the weight of this evidence can be questioned in at least two respects. First, it may be argued that our measure could fail to reflect expectancy of E2 in the same manner that RTs reflect automatic activation. Indeed, participants were allowed to move the slider at any moment during the session, and it is possible that its current position at E1 onset was in fact left unchanged during the E1-E2 interval. In that case, our score would reflect expectancy of E2 prior to the onset of E1, whereas RTs measured the automatic activation elicited by E1. Such a possibility is made more likely by (a) the fact that the interval between the onset of E1 and the onset of E2 was only $500 \mathrm{~ms}$ and (b) the fact that participants might have found it difficult to move the slider with their left hand while preparing to respond as quickly as possible to the signal of response with their right hand. 
A second potential problem concerns the RT pattern we obtained. Although we observed the same trend as those reported by Perruchet (1985; see also Clark et al., 2001) for eyeblink conditioning, our results, though significant, are nevertheless not as clear cut. This discrepancy may result from a genuine difference between RTs and conditioning procedures. However, it is also possible that asking participants to provide online ratings of their subjective expectancy interfered with the RT task.

These two limitations of our findings actually originate in a single aspect of our design, namely the concurrent measures of expectancy and RTs. Simultaneous collection of measures of expectancy and of measures of priming might actually be damaging for the proper assessment of each. In the following experiments, we therefore measured each on different groups of participants. In Experiment 2, participants were asked to report their expectancies. We implemented a few procedural changes to ensure that expectancies were indeed expressed only during the E1-E2 interval. Experiments 3 and 4 involved exactly the same pattern of events and the same timing, but participants were now simply asked to respond to the target as quickly as possible. This strategy allowed us to assess expectancy and RTs at the very same time during the procedure, although, of course, over different experiments. We return to the limitations of this research strategy in the General Discussion.

\section{Experiment 2}

In Experiment 2, participants were no longer asked to respond to the white square. Their only task consisted of expressing the extent to which they expected the white square to appear on each trial. This experiment followed the same design as Experiment 1, except for a few methodological changes aimed at increasing the validity of our measure of subjective expectancy. Participants now had to make their predictions after the onset of E1. To ensure that participants followed these instructions, we set the location of the cursor on the screen to zero at the onset of each trial, and it could only be moved after the onset of the tone, that is, during the interval between $\mathrm{E} 1$ and $\mathrm{E} 2$.

\section{Method}

Participants. Twenty-two participants, none of whom had participated in Experiment 1, were recruited from the same population to take part in Experiment 2.

Materials. The materials and design were identical to those of Experiment 1 , with the following exceptions. The cursor was now set in the middle location of the rating scale on each trial. This procedure prevented the use of a potentiometer to express expectancies (this would have implied that participants reset the slider of the potentiometer in medial location). Instead, participants pressed either the right or the left arrows of the keyboard according to the direction they wished to displace the cursor on the screen. The continuous scale was replaced by a 5-point scale. This scale appeared at the bottom of the screen at the tone onset and disappeared at the tone offset. The right end of the scale was marked "+" and the left end of the scale was marked "-."

The white square was then displayed at a fixed location at the center of the screen, where it replaced an otherwise permanently visible fixation point (a 1- $\times 1-\mathrm{cm}$ cross) on relevant trials.

Procedure. Participants were told that a white square would follow a tone on half of the trials. They were instructed to express their current expectancy for the occurrence of the square after each tone by using the arrow keys of the computer keyboard. They were told that the left endpoint indicated that they were certain that the square would not occur and that the right endpoint indicated that they were certain that the square would occur on that trial. Finally, participants were also told that they could use the two intermediate positions to indicate weaker confidence in their predictions. Participants were not required to move the cursor on each trial, but they were encouraged to do so as often as possible. To familiarize participants with this setup, they performed five preliminary trials prior to the onset of the experiment. On each such trials participants were asked to move the cursor alternatively on both sides.

Other aspects of the procedure were identical to those of Experiment 1, except that the tone-square SOA was set to $750 \mathrm{~ms}$ instead of $500 \mathrm{~ms}$. This value was chosen so as to strike a balance between the constraints set by two opposite requirements. The first was to make the interval long enough to make it possible for participants to move the cursor indicating their expectancies. The second was to keep the interval short enough to make it possible for us to capture the automatic activation elicited by E1 in further RT experiments involving the same parameter. Finally, the average ITI was shortened from 10 to $8 \mathrm{~s}$ (range $=6-10 \mathrm{~s}$ ).

Data analysis. The location of the cursor was collected $900 \mathrm{~ms}$ after onset of the tone. Because the SOA was $750 \mathrm{~ms}$, this means that there was a 150-ms overlap between the interval available to express expectancy and the display of the white square on the E1-E2 trials. This overlap made it possible to measure expectancy at a point time that was as close as possible to the onset of the white square while allowing for movement latency. It could be argued that some participants are fast enough to modify the location of the cursor on the expectancy scale during the 150 ms overlap as a function of whether the white square has occurred. This possibility can hardly be ruled out a priori, because the exact value of the minimum movement latency varies and is difficult to evaluate for specific experimental situations (Schmidt, 1988). However, it is worth noting that, in that case, the expectancy ratings would simply reflect the distribution of the white squares across trials. Because the occurrence of the white square was, by construction, independent from the context set by prior trials, expectancy ratings would also be independent from this context. In other words, one would not find a gambler's fallacy pattern in the data but rather a flat curve.

Finally, in this and in subsequent experiments, we pooled performance over runs of four and five trials to overcome a limitation of the original design, namely that there is only one response per participant after a run of length five trials and two after runs of length four trials.

\section{Results and Discussion}

Mean expectancies are plotted in Figure 3. They differed as a function of the length and nature of the preceding run of trials, $F(7$, $147)=11.43, p<.001, M S E=0.37$, with the strongest expectancies being observed after the longest run of tone-alone trials, and the lowest expectancies occurring after the longest run of tonesquare pairings. The linear trend was significant, $F(1,21)=8.59$, $p=.008, M S E=1.29$, with a negative slope. However, a strong positive recency effect was also apparent. Accordingly, the quadratic and cubic components were also significant, $F(1,21)=7.17$, $p=.015, M S E=0.35$, and $F(1,21)=37.35, p<.001, M S E=$ 0.27 , respectively.

The positive recency effect, whereby expectancy of the target after a single tone-alone event is lower than after a single tonesquare pairing, was much stronger than in Experiment 1. There were several differences between the two experiments: The use of a 5-point rating scale instead of a continuous scale, expression of expectancies after the tone onset, use of the keyboard instead of a potentiometer, suppression of the concurrent RT task, and longer tone-square SOA. It is not clear which of these differences may 


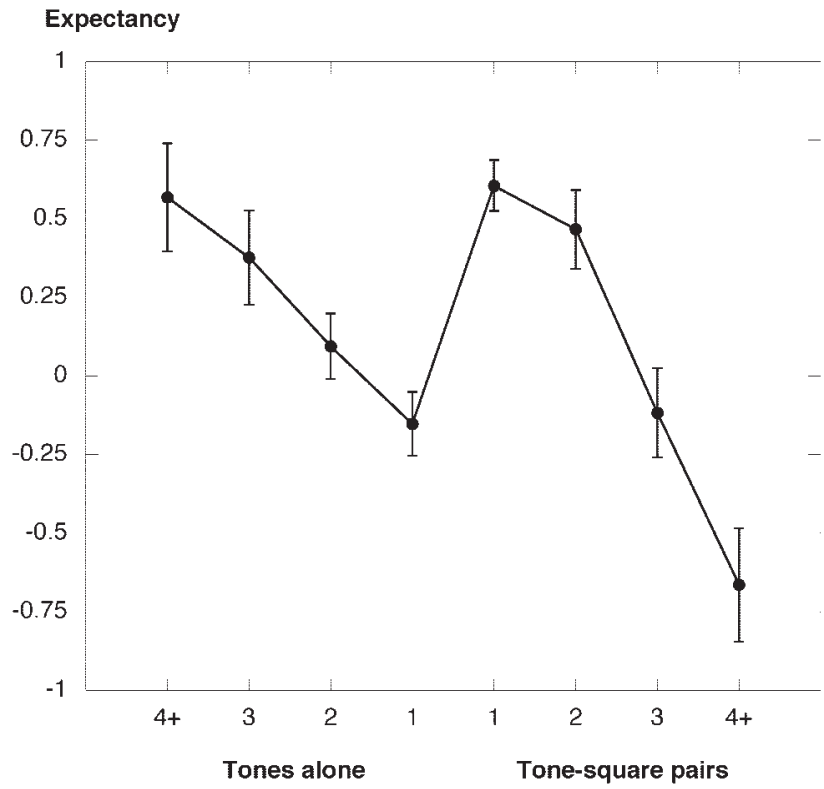

Figure 3. Mean conscious expectancy ratings (on a 5-point scale) as a function of the preceding run of trials (Experiment 2). The errors bars indicate standard errors.

have induced the observed pattern. Irrespective of the cause, we believe that the pattern we obtained here actually provides a better picture of the true variation of expectancies than the nearly linear pattern observed in Experiment 1. Indeed, the conditions involved here were intended to promote a more reliable measure of expectancy. In addition, a positive recency effect has been frequently observed in similar conditions (e.g., Hyman, 1953).

In the following experiments, we report RT linear trends, as in Experiment 1. However, to make it possible to examine the data while removing the possible influence of a positive recency effect for short runs, we also contrast runs consisting of three or more tone-alone trials with runs consisting of three or more tone-square pairings. Indeed, Figure 3 shows there was no overlap in the level of expectancies for those extreme values. Unsurprisingly, the difference was statistically reliable, $F(1,21)=15.66, p<.001$, $M S E=1.05$.

\section{Experiment 3}

Experiment 3 followed the same design as Experiment 2, except that participants were now asked to react to the appearance of the white square as quickly as possible, instead of providing expectancy ratings.

\section{Method}

Participants. Twenty participants, none of whom had participated in previous experiments, were recruited from the same population to take part in Experiment 3.

Materials and procedure. They were exactly the same as in Experiment 2, except that, as in Experiment 1, participants were asked to press the space bar of the keyboard with their right hand as quickly as possible following the appearance of the square.
Data analysis. Given the simplicity of the task, only one RT fell outside of the range defined for Experiment 1. This single RT value was replaced by the participant's mean RT.

\section{Results and Discussion}

RTs are plotted in Figure 4. It can be seen that overall, RTs were shorter than in Experiment 1. Average RTs differed as a function of the length and nature of the preceding run of trials, $F(7,133)=$ 3.62, $p=.001, M S E=438.93$, and the linear trend was again significant, $F(1,19)=10.92, p=.0037, M S E=899.62$. When pooled, the other polynomial components (quadratic, cubic, and so on) were no longer significant, $F(6,114)=0.601, p=.729$, $M S E=362.14$. RTs now followed a nearly perfect straight line with a negative slope, with the slowest RTs to the visual signal being observed after the longest run of tone-alone trials and the shortest RTs occurring after the longest run of tone-square pairings. Unsurprisingly, planned comparisons showed that the difference between RTs for the runs comprising three or more tonealone trials and RTs for the runs comprising three or more tonesquare pairings was significant, $F(1,19)=9.90, p=.005, M S E=$ 803.41.

Although these results are strongly suggestive that automatic associative activation prevails over conscious expectancy, it could be argued that the associative nature of the phenomenon has not been yet clearly ascertained. In the introductory section, we described the repetition effect (e.g., Bertelson, 1961), according to which the speed of responding is faster for repetitions than for alternations in random serial RT tasks with short RSIs. The facilitatory effect of repetition changes into an alternation effect with longer RSIs. These effects appear as potential confounding factors, because the succession of runs of E1-alone and E1-E2 pairings in our design is partially confounded with the distribution of the

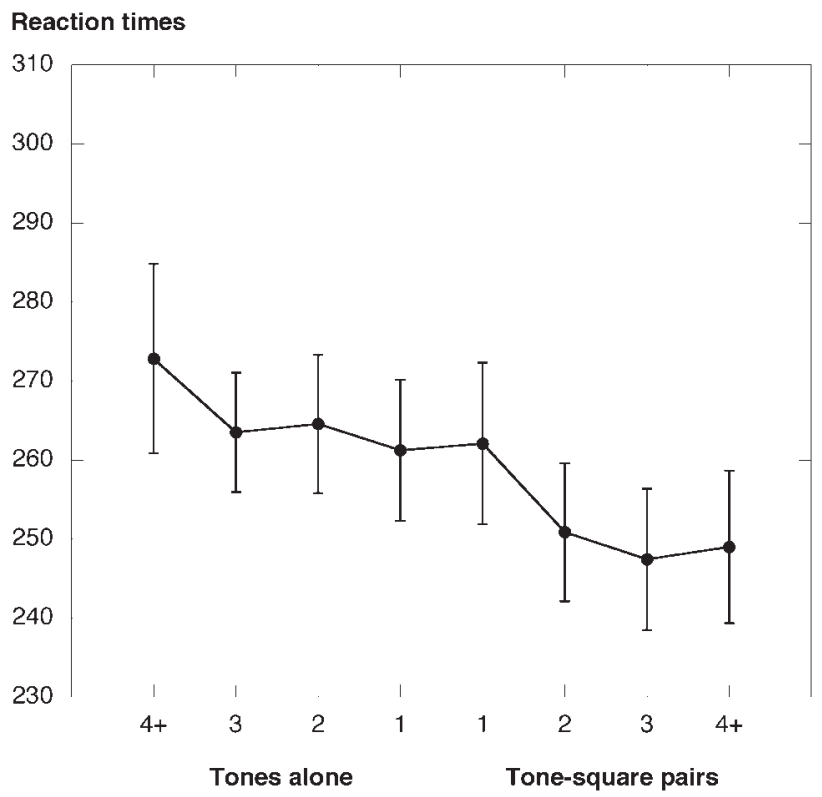

Figure 4. Mean reaction times (in milliseconds) to the second event (E2) as a function of the preceding run of trials (Experiment 3). The errors bars indicate standard errors. 

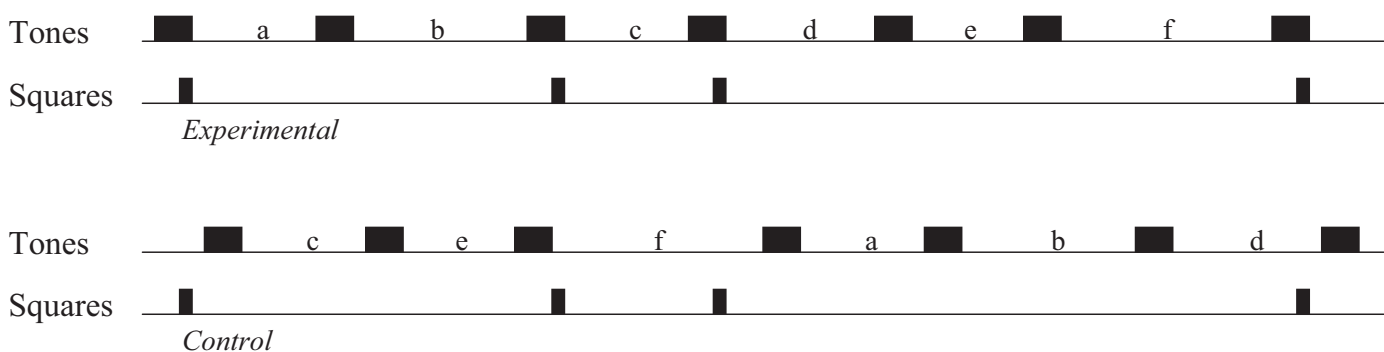

Figure 5. Participants from the experimental and from the control groups were yoked in such a way that the 2 participants from each pair were exposed to the very same sequence of squares (the response signal). The number of tones, as well as the distribution of the between-tones intervals, were also identical. However, the order of those intervals, labeled $a, b, c, d, e$, and $f$, for the experimental participant in the figure, was randomized for his or her control counterpart (e.g., $c, e, f, a, b$, and $d$ ). This procedure ensured that experimental and control participants were exposed to sequences of events as similar as possible, the only difference being that the temporal relation between tones and squares was broken for control participants (note that for the sake of simplicity, the figure illustrates the randomization procedure on a subset of the whole set of trials).

temporal intervals between successive E2 events. Although our paradigm was designed to prevent the occurrence of repetition priming, notably by using an ITI far longer than the intervals in which this effect has been observed in prior studies, the possibility that the present pattern of results originates in nonassociative factors warrants further examination. Exploring this possibility was the main goal of Experiment 4.

\section{Experiment 4}

The problem of ascertaining the associative nature of learning has been extensively investigated in conditioning research. Indeed, any conditioning session involves the repetition of CS-US pairs, and it is crucial in such settings that changes in performance may be unambiguously ascribed to the experience of the pairings per se, and not merely to repetition of the CS or the US. Fortunately, this problem can be addressed in a straightforward manner that involves comparing performance in the standard experimental condition with performance in a condition in which the CS and US occur without being paired. Such a control was used by Perruchet (1985). The absence of a run length effect in this control condition is precisely what made it possible to conclude that the effect obtained in the experimental condition was due to associative factors.

Experiment 4 was designed to implement a similar control for the RT paradigm used in Experiments 1 and 3. In Experiment 4, one group of participants was submitted to E1-E2 pairings in the very same conditions as those used in Experiment 3, so as to replicate the observed phenomenon on another sample of participants. A second group of participants was exposed to the same number of E1 and E2 events as the first group was, but E1 and E2 were no longer paired. Because the crucial factor was the temporal distribution of E2 events, this distribution was identical for the two groups. To fulfill this constraint while retaining the individual parameter randomization implemented in the prior experiments, participants were yoked in such a way that each control participant was exposed to exactly the same sequence of E2 events as a unique, randomly assigned experimental participant. Any 2 yoked participants were also exposed to sequences of E1 events that followed the same overall distribution of temporal intervals ${ }^{3}$, but the order of the intervals between successive E1 events observed for the experimental participant was randomized for the yoked control participants. This meant that E1 and E2 occurred independently from each other in the control condition-a design known as a "truly random" control in the conditioning literature (Rescorla, 1967). If the run effect we obtained in Experiments 1 and 2 was due to the temporal distribution of specific events independent of their relationships, it should also occur in the control group.

\section{Method}

Forty new participants drawn from the same population as for the previous experiments took part in Experiment 4. For the experimental group $(n=20)$, the conditions exactly replicated those used in Experiment 3 . For the control group $(n=20)$, participants were yoked in such a way that within each pair of participants, a participant from the control group was shown the very same sequence of E2 events as his or her experimental counterpart. As illustrated in Figure 5, the distribution of the intervals between successive E1 events was collected for each participant of the experimental group and then applied after randomization to his or her yoked participant of the control group.

\section{Results and Discussion}

Results are shown in Figure 6, plotted separately for the experimental and control conditions. Figure 6 also shows (right $y$-axis) the difference between the two groups for each run length. RTs were submitted to a 2 (groups) $\times 8$ (runs) ANOVA with repeated measures on the last factor. The main effect of group was significant, $F(1,38)=102.58, p<.001, M S E=14,247.33$, and

\footnotetext{
${ }^{3}$ It could be argued that, unlike conditioning situations, the repetition of E1 is not crucial and that E1 could have been simply removed from the design. It remains, however, that removing E1 would have deeply altered the nature of the task required from the participants. Indeed, given that the white square would in this situation be the only stimulus, participants would have had to press a key in response to each and all events that occur in the session. In contrast, in the experimental group of Experiment 3 , participants still had to make a choice in that they had to respond to the white square but not to the tone. It seems advisable to retain this aspect of the task in both experimental and control conditions.
} 


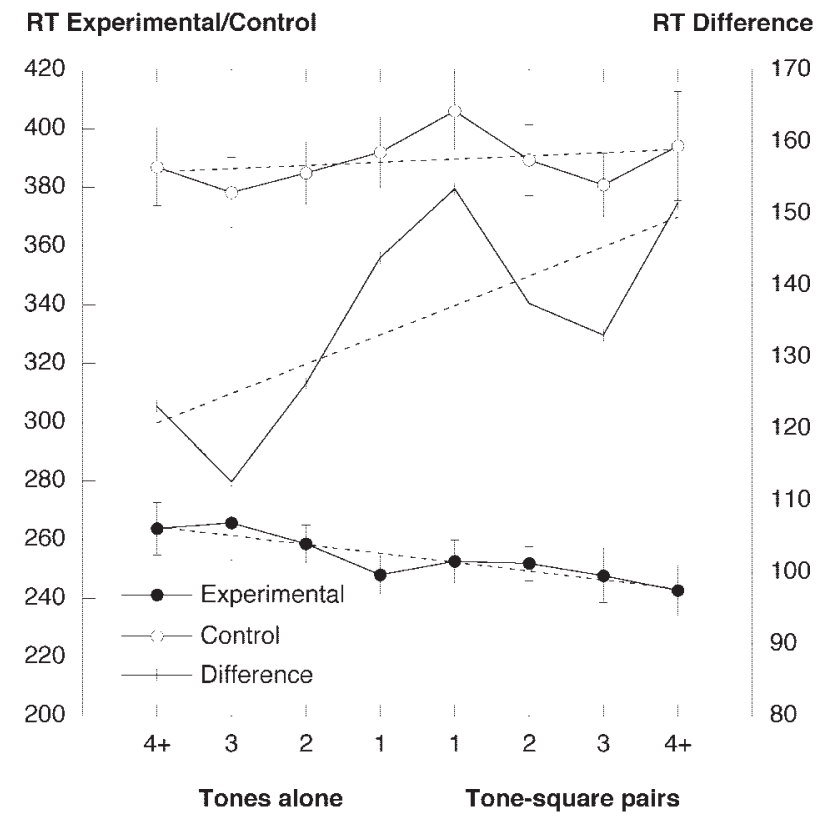

Figure 6. Mean reaction times (in milliseconds) to E2 as a function of the preceding run of trials, for the experimental group and for a control group (left $y$-axis) in which tones and squares were unpaired (Experiment 4). The figure also shows (right $y$-axis) the mean reaction time difference between the two groups. Note that for the control group, the indicated values are the values collected for the trials that correspond to the labels of the $x$-axis for the experimental group. The errors bars indicate standard errors.

indicates that the presence of a preparatory signal results in a facilitation of $135.2 \mathrm{~ms}$. The main effect of runs was not significant, $F(7,266)=1.13, p=.34, M S E=714.38$. However, there was a significant Group $\times$ Runs interaction, $F(7,266)=2.84, p=$ $.0072, M S E=714.38$. The interaction between the linear trends associated with the RT distribution of each of the two groups was also significant, $F(1,38)=4.58, p=.0387, M S E=1,534.88$, indicating that the slopes of the regression line characterizing how RT changes with run length were different in each group. For the experimental group, the slope of the regression line was negative and significantly different from $0, F(1,38)=4.97, p=.032$, $M S E=1,534.88$. In contrast, the slope of the regression line for the control group was positive, although nonsignificantly so, $F(1$, 38) $=0.64, p=.429, M S E=1,534.88$. These results were confirmed by planned comparison analyses comparing performance on the long runs of trials. The difference between RTs for runs comprising three or more tone-alone trials and for the runs comprising three or more tone-square pairings was significant for the experimental group, $F(1,38)=5.16, p=.029, M S E=$ $1,477.88$, but nearly null for the control group $(5 \mathrm{~ms}$ in the reversed direction) and not significant, $F(1,38)=0.32, p=.573, M S E=$ $1,477.88$.

These results therefore replicate our previous findings: The slowest RTs to the visual signal were observed after the longest run of E1-alone events, and the shortest RTs were observed after the longest run of E1-E2 pairings. Given that (a) the intervals between successive E2 events were exactly identical for the experimental and control groups and (b) performance for the control group was roughly flat, this effect cannot be attributed to the temporal distribution of E2 events. These results are therefore strongly indicative that associative activation is specifically responsible for the performance of the experimental group.

\section{General Discussion}

The four experiments reported herein are based on a paradigm that makes it possible to contrast the effects of two putative mechanisms widely assumed to account for improved processing of an event (E2) when this event has been repeatedly paired with another (E1): conscious expectancy for E2 upon presentation of $\mathrm{E} 1$, on the one hand, and automatic activation resulting from repeated associations between $\mathrm{E} 1$ and $\mathrm{E} 2$, on the other hand.

In Experiment 1, participants were asked to provide both an expectancy judgment on each trial and a speeded response to E2 when E2 occurred. Results confirmed that conscious expectancy for E2 followed the gambler's fallacy (e.g., Burns \& Corpus, 2004; Jarvik, 1951) in the conditions we explored. The data also suggested that RTs follow a completely opposite pattern in the very same conditions. However, the fact that participants were engaged in a dual task may have been detrimental for both measures. Whether participants actually updated their rating of expectancy upon presentation of E1 remained somewhat doubtful, and the pattern of RTs was not as clear cut as in the eyeblink conditioning situation described by Perruchet (1985). Therefore, in subsequent experiments, participants were asked either for expectancy rating or for speeded responses to E2.

In Experiment 2, participants were asked to report their expectancy for E2 upon presentation of E1, and the results again provided strong evidence for the influence of the gambler's fallacy, at least when runs involving more than two trials were considered (a positive recency effect was observed for shorter runs). In Experiment 3, participants were simply asked to respond as quickly as possible to E2. We found that the shortest RTs were obtained on precisely those trials associated with minimal expectancy over Experiments 1 and 2 and, conversely, that the longest RTs occurred on those trials for which expectancy had been maximal in Experiments 1 and 2 .

Arguably, the observed distribution of RTs could be due to a transient aftereffect of stimulus presentation or of response production-that is, processes that do not depend on the association between E1 and E2. This possibility finds support in the beneficial effect of immediate repetition that occurs with short ITIs in random serial RT tasks (e.g., Bertelson, 1961). Although our experimental conditions had been designed to prevent the occurrence of repetition priming (particularly through the use of very long ITIs), we nevertheless conducted an additional experiment so as to definitely rule out this and any other account based on nonassociative mechanisms. Thus, in Experiment 4, control participants were exposed to the same distribution of E2 events as experimental participants, but the occurrence of E1 events was random. Under those conditions, RTs did not vary significantly as a function of the preceding runs, whereas the effect observed in the prior experiments was successfully replicated for the experimental group. We therefore conclude that the main determinant of RT in this situation is the strength of the association between E1 and E2 events.

It could be argued that our conclusions are mitigated by the fact that most of our results are based on experiments in which expect- 
ancies and RTs had been collected separately. Thus, actual subjective expectancy could differ between Experiments 1 and 2, in which participants were asked to make their judgments explicit, and Experiments 3 and 4, in which no expectancy rating was required. It should be noted, however, that asking for keypressing and expectancy judgments at the very same moment in the same participants is not possible. Thus the choice is between collecting both measures almost simultaneously, as in Experiment 1-which entails risks of invalidating both measures because of the simultaneity requirement-or asking participants to perform the tasks separately. We chose the latter option for Experiments 2-4 because it makes it possible to compare expectancy ratings and RTs exactly at the same moment after the tone onset-a condition that seemed essential in the present context. It is worth adding that to rule out our interpretation of the data, it would be necessary to assume not only that expectancy differs quantitatively as a function of whether it is expressed through a rating or not, but also that it completely reverses from one condition to the other. This appears somewhat implausible.

However, it remains that it would be useful to confirm our findings in conditions that overcome any objection based on the fact that RTs and expectancies were collected separately. A possible strategy for further studies would consist of using some trials as a probe for assessing explicit expectancies in an otherwise standard RT paradigm. In this design, participants would be asked to rate their expectancy instead of pressing the response key whenever a trial is preceded by a predefined signal. A foreseeable difficulty with this strategy is that because the most informative trials are those that follow the longest runs, they are also the less frequent. Asking for an expectancy judgment on those crucial trials would further reduce the number of observations on which RTs are measured. A different solution, one that would not suffer from this limitation, would be to assess expectancy using event-related potentials instead of explicit ratings. An obvious candidate for this objective is the contingent negative variation, which is a negative deflection elicited between a preparatory signal and the response signal in a RT paradigm. The late component of the contingent negative variation is thought to reflect anticipation of the response signal. Instead of using an expectation-related potential, a further possibility would consist of measuring the event-related potential components evoked by an unexpected event. Of special interest here is the so-called P300 component, which is a large positive wave that occurs approximately $300 \mathrm{~ms}$ after the onset of an unexpected stimulus (for a review, see Polich \& Kok, 1995). Note that the P300 should be elicited not only when the response signal occurs unexpectedly (presumably after a long run of tone-square pairs), but also when the response signal fails to occur when it is expected (the so-called omission-evoked potentials, which would presumably occur after a long run of tones alone).

Another potential argument against the significance of our results could be that the changes in RT we observed over the different conditions were generally moderate in size. Taking into account runs involving three or more trials (i.e. those for which expectancies clearly followed the gambler's fallacy), the differences between RTs to stimuli occurring after runs of E1-alone trials and after runs of E1-E2 pairings were $21.3 \mathrm{~ms}, 19.9 \mathrm{~ms}$, and $19.5 \mathrm{~ms}$ for Experiments 1, 3, and 4 respectively. When expressed as Cohen's $d$ (e.g., Cohen, 1988), these effects fall into the range of medium size effects according to his conventions (respectively, $d s=.529, .494$, and .473). However, it is important to bear in mind that automatic activation was pitted against conscious expectancy in our paradigm and, hence, that the moderate changes we observed presumably resulted from two opposite sources of influence. Our design, by construction, was therefore not optimal with respect to assessing how strong an influence automatic activation can exert independent of other factors; however, the very fact that we observed significant, if only moderate, activation even under these suboptimal conditions suggests that its influence was strong enough to overcome the influence of conscious expectancy.

Note also that this design limitation does not undermine the interest of our study, because our goal was only to document the existence of a mechanism that can account for the associative influence of E1 on the processing of E2 independent of the conscious expectancy elicited by E1. In this respect, our results are clear cut. They clearly generalize the eye-blink results previously described by Perruchet (1985) and Clark et al. (2001) to the domain of voluntary responses. Our results strengthen the conclusions of studies showing that RTs may follow the same general laws as classically conditioned reactions (Los \& Heslenfeld, 2005; Los, Knol, \& Boers, 2001; Los \& Van Den Heuvel, 2001; Razran, 1936; Rexroad, 1936; Stephens, 1937).

In the following, we first discuss why we think our claim for the existence of two independent processes is particularly straightforward in this situation. Next, we speculate on the nature of these processes.

The validity of inferring the existence of separable and independent processes based on the observation of dissociations has long been debated (Dunn \& Kirsner, 1988; Farah, 1994; Shallice, 1988). For instance, Kinder and Shanks (2003) showed how the dissociations between priming and recognition exhibited by amnesic patients can in fact be accounted for by a single-system connectionist model and are thus insufficient to establish the existence of separable memory systems. The same argument was put forward by Plaut and Shallice (1993), who showed that lesioning a single connectionist network in various ways could account for dissociations observed with patients.

Given the demonstrated limits of dissociation methodology, one could ask what makes us think that our data provide a compelling case for the existence of separable processes. In the terminology of Dunn and Kirsner (1988), our data provide evidence for a crossed double dissociation (also termed a "cross-over dissociation" after Shallice, 1988). Indeed, the same variable, namely the nature of the preceding run of trials, has opposite effects on two dependent variables, namely expectancy ratings and RTs. Shallice (1988) has argued that inferences from such a pattern of data are more valid than when the double dissociation is not crossed. However, as emphasized by Dunn and Kirsner, even the existence of a crossed double dissociation is not evidence for separate processes. It is still possible that the two variables under consideration depend in opposite ways on the same underlying process. To borrow a trivial example from Dunn and Kirsner's article: A cross-over dissociation between recall and recognition will be obtained if recall is assessed as the proportion of recalled items and recognition is measured as the proportion of errors (rather than proportion correct).

Such a limit is not insurmountable, however. The problem of inferring separable underlying processes stems from the fact that the two dependent variables may be a priori negatively correlated, 
as are the proportion of recall and the proportion of errors in recognition. Crucially, Dunn and Kirsner (1988) offered a strategy to settle the issue by suggesting that the influence of two independent processes is unquestionably established when one obtains evidence for what they dubbed "reversed association." A reversed association is defined "as the conjunction of a negative association (i.e. a cross-over dissociation) and a positive association" (Dunn \& Kirsner, 2003, p. 3). To quote again, "If one study finds that two tasks are affected in the same way by one or more variables and a different study finds them to be affected in opposite ways for one or more other variables, the results of the two studies together established a reversed association" (Dunn \& Kirsner, 1988, p.100). The dissociation between recall and recognition described above cannot provide a reversed association, in so far as there is no variable that would increase both the proportion of correct responses in recall and the proportion of errors in recognition. By contrast, and returning now to our own results, we have obtained a cross-over dissociation while, crucially, positive associations between expectancy and RTs (whereby increased expectancy is linked to RTs improvement) are well-documented in a large amount of earlier studies (e.g., Niemi \& Näätänen, 1981). To the best of our knowledge, our results, when considered together with the previous studies documenting associations between expectancy and voluntary responses, therefore provide the first demonstration for a reversed association in this research context. We therefore conclude, with fairly good confidence, that the associative effects of E1 on E2 (i.e., those effects ascribable to the repeated E1-E2 pairings) stem from the influence of at least two independent processes.

Of course, the existence of a reversed association is in and of itself mute with respect to the nature of the processes involved. The nature of our manipulation makes it reasonably straightforward to surmise that the two processes at hand can be respectively labeled as conscious expectancy and automatic associative activation. The meaning of these labels needs to be specified, however, because they are potentially misguiding. A longstanding debate concerns the role of consciousness in learning. In the domain of conditioning, several authors have argued that there is currently no evidence for learning in the absence of participants' conscious awareness of the relationships between the conditioned and the unconditioned stimuli (e.g., Brewer, 1974; Lovibond \& Shanks, 2002). Likewise, one of the main areas of debate in the growing body of research dedicated to implicit learning (for a review, see Cleeremans, Destrebecqz, \& Boyer, 1998) lies around the possibility of learning about structured material without conscious representation of the structural relationships. This possibility has been denied by a number of contributors (e.g., Perruchet \& Vinter, 2002; Shanks \& St. John, 1994). The present evidence for an effect of automatic associative activation could be taken as a counterargument against this standpoint. It is worth emphasizing that such a conclusion would be unwarranted (see Los \& Van Den Heuvel, 2001, p. 373, for a similar judgment). Our results do not provide fuel to those who argue for the existence of unconscious forms of conditioning (e.g., Clark, Manns, \& Squire, 2002) or unconscious learning in complex experimental settings (e.g., Reber, 1993). Indeed, in our situation, participants were not prevented from paying attention to the stimuli, and moreover, they were informed about the crucial relationships before the experimental session began.

Our demonstration instead concerns the existence of mechanisms capable of facilitating voluntary reactions to E2 independently from conscious expectancy of E2. The only claim that can be made about these mechanisms based on our results is that they follow laws of associative learning that have been identified for reflexive behavior during the behaviorist era, namely: They are strengthened by the repetition of E1-E2 associations and extinguished by the presentation of E1 alone.

Further studies will have to be dedicated to exploring in a more thorough manner the nature of these mechanisms and their onset conditions. A promising strategy concerns the search for the conditions in which the expression of these processes is favored or hampered. In eyeblink conditioning, Clark et al. (2001) reported that the dissociation between subjective expectancy and eyeblink responses disappeared and even turned into an association when the tone terminated before the onset of the air puff (a preparation known as "trace conditioning") instead of coterminating with E2 (a preparation known as "delay conditioning"). The reliability of this pattern of data has been questioned. Indeed, Shanks and Lovibond (2002) noted that the association between expectancy and eyeblink responses that Clark et al. reported to have obtained in trace conditioning was due to a very small number of measures and reached significance only when the data were analyzed in a specific manner. Irrespective of the reliability of the phenomenon in eyeblink conditioning however, whether RTs patterns would exhibit an inversion when the prime no longer overlaps the target remains an open question.

Another question that warrants exploration is suggested by the results obtained by Alegria (1978), according to which the RT pattern that we have obtained in the present study in a simple RT task could also be observed in a dual-choice RT task. In other words, the RT pattern could persist even though the same preparatory signal may precede two different response signals. Alegria's results were based on only 6 participants, and the temporal parameters of his experiment (notably the short ITIs) made it possible that the RT pattern was at least partially due to simple repetition priming (e.g., Bertelson, 1961). If the phenomenon suggested by Alegria turns out to be empirically reliable, it will be worth examining whether it is compatible with the interpretation we have proposed for our own results, which relies heavily on basic laws of associative learning. The response is far from being obvious, because to our knowledge, Alegria's paradigm has never been implemented in the traditional literature on conditioning, in which a given conditioned stimulus is typically associated with a single unconditioned stimulus.

\section{References}

Alegria, J. (1978). Sequential effects of catch-trials on choice reaction time. Acta Psychologica, 42, 1-6.

Anderson, J. R. (2000). Learning and memory (2nd ed.). New York: Wiley. Anderson, N. H. (1960). Effect of first-order conditional probability in a twochoice learning situation. Journal of Experimental Psychology, 56, 73-93.

Bertelson, P. (1961). Sequential redundancy and speed in a serial twochoice responding task. Quarterly Journal of Experimental Psychology, 13, 90-102.

Bertelson, P. (1963). S-R relationships and reaction times to new versus repeated signals in a serial task. Journal of Experimental Psychology, 65, $478-484$.

Bertelson, P., \& Renkin, A. (1966). Reaction times to new versus repeated signals in a serial task as a function of response-signal time interval. Acta Psychologica, 25, 132-136.

Bolles, R. C. (1972). Reinforcement, expectancy, and learning. Psychological Review, 79, 394-409. 
Bonic, I. (1989). A test for an expectancy theory of human eyeblink classical conditioning. Unpublished honors thesis, University of New South Wales, Australia.

Brewer, W. F. (1974). There is no convincing evidence for operant or classical conditioning in adult humans. In W. Weiner \& D. Palermo (Eds.), Cognition and symbolic processes (pp. 1-42). Hillsdale, NJ: Erlbaum.

Burns, B. D., \& Corpus, B. (2004). Randomness and inductions from streaks: "Gambler's fallacy" versus "Hot hand." Psychonomic Bulletin and Review, 11, 179-184.

Bush, R. R., \& Mosteller, F. A. (1951). A model for stimulus generalization and discrimination. Psychological Review, 58, 413-423.

Clark, R. E., Manns, J. R., \& Squire, L. R. (2001). Trace and delay eyeblink conditioning: Contrasting phenomena of declarative and nondeclarative memory. Psychological Science, 12, 304-308.

Clark, R. E., Manns, J. R., \& Squire, L. R. (2002). Classical conditioning, awareness, and brain systems. Trends in Cognitive Sciences, 6, 524-531.

Cleeremans, A., Destrebecqz, A., \& Boyer, M. (1998). Implicit learning: News from the front. Trends in Cognitive Sciences, 2, 406-416.

Cohen, J. (1988). Statistical power analysis for the behavioral sciences (2nd ed.). Hillsdale, NJ: Erlbaum.

Dunn, J. C., \& Kirsner, K. (1988). Discovering functionally independent mental process: The principle of reversed association. Psychological Review, 95, 91-101.

Dunn, J. C., \& Kirsner, K. (2003). What can we infer from double dissociations? Cortex, 39, 1-7.

Epstein, S., \& Roupenian, A. (1970). Heart rate and skin conductance during experimentally induced anxiety: The effect of uncertainty about receiving a noxious stimulus. Journal of Personality and Social Psychology, 16, 20-28.

Farah, M. J. (1994). Neuropsychological inference with an interactive brain: A critique of the "locality" assumption. Behavioral and Brain Sciences, 17, 43-104.

Hale, D. J. (1967). Sequential effects in a two-choice serial reaction task. Quarterly Journal of Experimental Psychology, 19, 133-141.

Hull, C. L. (1943). Principles of behavior. New York: Appleton-Century-Crofts.

Hyman, R. (1953). Stimulus information as a determinant of reaction time. Journal of Experimental Psychology, 45, 188-196.

Jarvik, M. E. (1951). Probability learning and a negative recency effect in the serial anticipation of alternative symbols. Journal of Experimental Psychology, 41, 291-297.

Keren, G., \& Lewis, C. (1994). The two fallacies of gamblers: Type I and Type II. Organizational Behavior and Human Decision Processes, 60, $75-89$.

Kinder, A., \& Shanks, D. A. (2003). Neuropsychological dissociations between priming and recognition: A single-system connectionist account. Psychological Review, 110, 728-744.

Knight, D. C., Nguyen, H. T., \& Bandettini, P. A. (2003). Expression of conditional fear with and without awareness. Proceedings of the National Academy of Sciences, 100, 15280-15283.

Kornblum, S. (1973). Sequential effects in choice RT: A tutorial review. In S. Kornblum (Ed.), Attention and performance IV (pp. 259-288). New York: Academic Press.

Los, S. A., \& Heslenfeld, D. J. (2005). Intentional and unintentional contributions to nonspecific preparation: Electrophysiological evidence. Journal of Experimental Psychology: General, 134, 52-72.

Los, S. A., Knol, D. L., \& Boers, R. M. (2001). The foreperiod effect revisited: Conditioning as a basis for nonspecific preparation. Acta Psychologica, 106, 121-145.

Los, S. A., \& Van Den Heuvel, C. E. (2001). Intentional and unintentional contributions to nonspecific preparation during reaction time foreperiods. Journal of Experimental Psychology: Human Perception and Performance, 27, 370-386.
Lovibond, P. F., \& Shanks, D. R. (2002). The role of awareness in Pavlovian conditioning: Empirical evidence and theoretical implications. Journal of Experimental Psychology: Animal Behavior Processes, 28, 3-26.

Matt, J., Leuthold, H., \& Sommer, W. (1992). Differential effects of voluntary expectancies on reaction times and event-related potentials: Evidence for automatic and controlled expectancies. Journal of Experimental Psychology: Learning, Memory, and Cognition, 18, 810-822.

Nicks, D. C. (1959). Prediction of sequential two-choice decisions from event runs. Journal of Experimental Psychology, 57, 105-114.

Niemi, P., \& Näätänen, R. (1981). Foreperiod and simple reaction time. Psychological Bulletin, 89, 133-162.

Perruchet, P. (1985). A pitfall for the expectancy theory of human eyelid conditioning. Pavlovian Journal of Biological Sciences, 20, 163-170.

Perruchet, P., \& Vinter, A. (2002). The self-organizing consciousness. Behavioral and Brain Sciences, 25, 297-388.

Plaut, D. C., \& Shallice, T. (1993). Deep dyslexia: A case study of connectionist neuropsychology. Cognitive Neuropsychology, 10, 377-500.

Polich, J., \& Kok, A. (1995). Cognitive and biological determinants of P300: An integrative review. Biological Psychology, 41, 103-146.

Razran, G. H. S. (1936). Discussion: The conditioning of voluntary reactions. Journal of Experimental Psychology, 19, 654.

Reber, A. S. (1993). Implicit learning and tacit knowledge: An essay on the cognitive unconscious. New York: Oxford University Press.

Requin, J., Brener, J., \& Ring, C. (1991). Preparation for action. In J. R. Jennings \& M. G. H. Coles (Eds.), Handbook of cognitive psychophysiology: Central and autonomous nervous system approaches (pp. 357448). New York: Wiley.

Rescorla, R. A. (1967). Pavlovian conditioning and its proper control procedures. Psychological Review, 74, 71-80.

Rexroad, C. N. (1936). Reaction times and conditioning: First studies. Journal of Experimental Psychology, 19, 144-158.

Schmidt, R. A. (1988). Motor control and learning: A behavioral emphasis (2nd ed.). Champaign, IL: Human Kinetics.

Shallice, T. (1988). From neuropsychology to mental structure. Cambridge, England: Cambridge University Press.

Shanks, D. R., \& Lovibond, P. F. (2002). Autonomic and eyeblink conditioning are closely related to contingency awareness. Journal of Experimental Psychology: Animal Behavior Processes, 28, 38-42.

Shanks, D. R., \& St. John, M. F. (1994). Characteristics of dissociable human learning systems. Behavioral and Brain Sciences, 17, 367-447.

Smulders, S. F. A., Notebaert, W., Meijer, M., Crone, E. A., Van Der Molen, M. W., \& Soetens, E. (2005). Sequential effects on speeded information processing: A developmental study. Journal of Experimental Child Psychology, 90, 208-234.

Soetens, E., \& Notebaert, W. (2005). Response monitoring and expectancy in random serial RT tasks. Acta Psychologica, 119, 189-216.

Spence, C., Nicholls, M. E. R., \& Driver, J. (2001). The cost of expecting events in the wrong sensory modality. Perception and Psychophysics, 63, 330-336.

Stephens, J. M. (1937). Data bearing on the conditioning of voluntary reactions. Journal of Experimental Psychology, 21, 236-239.

Tolman, E. C. (1932). Purposive behavior in animal and man. New York, Appleton-Century-Crofts.

Vervaek, K. R., \& Boer, L. C. (1980). Sequential effects in two-choice reaction time: Subjective expectancy and automatic after-effect at short response-stimulus intervals. Acta Psychologica, 44, 175-190.

Received June 14, 2005

Revision received February 28, 2006

Accepted March 12, 2006 